Research paper

\title{
In-Silico Analysis of Natural Products that Modulates Enzymes of Diabetic Target
}

\author{
Babita Aryal ${ }^{1}$, Saroj Basnet ${ }^{2}$, Bishnu P. Marasini ${ }^{3}$, Karan Khadayat ${ }^{1}$, Darbin Kumar Poudel ${ }^{1}$, Ganesh \\ Lamichhane ${ }^{1}$, Prakriti Budhathoki ${ }^{1}$, Purushottam Niraula ${ }^{1}$, Sonika Dawadi ${ }^{1}$, Rishab Marahatha ${ }^{1}$, \\ Sitaram Phuyal ${ }^{1}$, and Niranjan Parajuli ${ }^{*}$
}

${ }^{1}$ Central Department of Chemistry, Tribhuvan University, Kirtipur, Kathmandu, Nepal

${ }^{2}$ Center for Drug Design \& Molecular Simulation Division, Cancer Care \& Research Center, Kathmandu, Nepal ${ }^{3}$ Department of Biotechnology, National College, Tribhuvan University, Naya Bazar, Kathmandu, Nepal *Correspondance: nparajuli@cdctu.edu.np, Tel: +977-1-4332034

\begin{abstract}
Metabolic enzymes are often targeted for drug development programs of metabolic diseases such as diabetes and its complications. Many secondary metabolites isolated from natural products have shown therapeutic action against these enzymes. However, some commercially available synthetic drugs have shown unfriendly impacts with various side effects. Thus, this research has focused on a comprehensive study of secondary metabolites showing better inhibitory activities towards metabolic enzymes such as $\alpha$-amylase, $\alpha$-glucosidase, aldose reductase, and lipase. Further receptor-based virtual screening was performed against the various secondary metabolites database designed in-silico. Using Gold combined with subsequent post-docking analyses, the score was obtained as methyl xestospongic ester (Gold score 65.83), 2,"4"-O-diacetylquercitrin (Gold score 65.15), kaempferol-3-O-neohesperidoside (Gold score 53.37) and isosalvianolic acid $C$ methyl ester (Gold score 53.44) for lipase, aldol reductase, $\alpha$-amylase, and $\alpha$-glucosidase, respectively. Besides, vitexin and isovitexin for $\alpha$-amylase; $\mathrm{N}$-trans-Caffeoyl-tyramin for $\alpha$-glucosidase; purpurolide $\mathrm{F}$ and schaftoside for lipase; acteoside and orientin for aldose reductase could be potential drugs for respective enzymes based on in-silico analyses, supported by experimental IC 50 values reported. They could bind to the competitive sites of the various targets of metabolic enzymes, and finally, toxicity analysis using ProTox-II was also performed.
\end{abstract}

Keywords: Enzyme inhibition, Secondary Metabolites, In-silico Analysis, Molecular docking, and Drug candidates

\section{Introduction}

Metabolic enzymes are key players in metabolism, and their abnormal secretion/function poses severe disorders causing distinctive pathogenesis in the body. Such metabolic disorders depend on the physiological conditions of the body and genetic factors as well; thus, the finding of such agents and understanding in pathways adds new dynamics to the field of pharmacology and biochemistry. Natural products have demonstrated diverse pharmacological effects because of the presence of secondary metabolites such as polyphenols, flavonoids, glycosides, alkaloids, etc. In developing nations, synthetic drugs are inaccessible as well as unreasonably expensive to the least fortunate 
population, so natural product derived drugs are, of course, better alternatives to treat diseases in the future. Some synthetic and semi-synthetic inhibitory drugs have shown unfriendly impacts with various side effects. It is argued that natural drugs are increasingly better over synthetic because of fewer side effects and better efficacy. Besides, nowadays, synthetic medication turns to natural products to prevent different diseases [1]-[3].

In the early days, people have been using crude extracts of natural products, which usually exhibit low efficacy due to the antagonistic effects of associate constituents. On the other hand, secondary metabolites have remarkably demonstrated potent enzyme inhibiting activity and will be more successful. Drug discovery programs based on crude extracts give only a baseline survey on the inhibitory potential of constituents, and results are not trustworthy as several factors influence the chemistry of metabolites. Thus, potent inhibitors isolated from crude extracts only could be drug candidates. As suggested, natural products or modified analog could reduce unfriendly symptoms [4].

Diabetes is one of the major global silent pandemics and affects about 463 million people; by 2045, this will ascend to 700 million and is among the top 10 causes of death [5], [6]. Diabetes causes life-limiting and life-threatening complications, to avoid these required long term clinical considerations either by diet or hypoglycemic agents that inhibit carbohydrate hydrolase enzyme [7]. Different classes of drugs are used for the regulation of blood glucose levels such as sulfonylureas, biguanides, meglitinide, thiazolidinediones etc [8]. However, due to the lack of specificity, these drugs are reported to cause several gastrointestinal side effects like cramping, flatulence, abdominal distention, and diarrhea [9].

Diabetic retinopathy is among the primary sources of vision misfortune identified with microvascular complications due to overexpression of aldose reductase and is the fifth driving reason for visual impairment and blindness around the world. According to WHO, at least 2.2 billion people have a vision impairment or blindness; among them, 3 million people have vision misfortune due to diabetic retinopathy. In a person with prolonged diabetes, aldose reductase is found to be sensitive in the cell that is insensitive to insulin. In other words, diabetic retinopathy is caused due to excess accumulation of sorbitol in the cell that is insensitive to insulin, i.e., retina. Sorbitol is thought to have multiple damaging effects in retinal capillaries, including osmotic damage [10], [11], and it can be controlled by inhibiting aldose reductase along with avoiding vision misfortune. Effective class of drugs includes cyclic imides derivatives (sorbinil, fidarestat), carboxylic acid derivatives (epalrestat, tolrestat, zenarestat, alrestatin). However, these drugs have detrimental side effects, such as hypersensitivity, hepatic dysfunction, and renal toxicity [12].

$39 \%$ of adults in the world are overweight and obese, which is accountable for excess triglyceride in the blood that not only causes liver and pancreas disease but also induces the risk of heart disease, which contributes $8 \%$ of death worldwide. Lipase is a key triglyceride hydrolase found in the luminal digestive tract and prompts the formation of absorbable monoglycerides. Lipases display a versatile subdomain called a lid, which controls the access of substrate molecules to the catalytic site [13]. The activity of lipase enhanced by bile salts and co-lipase protein. Absorbable mono-glycerides may abnormally accumulate in the tissues and organs because of a fatty diet and lack of physical 
fitness, causing overweight and obesity linked to various diseases, so lipase activity should be impeded. Some clinically approved lipase inhibitors are orlistat and cetilistat. A new class of inhibitor from nature is the anticipation and treatment of overweight and obese, attributable to the crucial job of lipase in lipid ingestion [14]. Thus, here we have performed molecular docking of possible drug candidates of natural products with metabolic enzymes (Figure 1S). We believe findings would be beneficial in drug development programs based on secondary metabolites.

\section{Results}

In this article Table $1 S$ provides details about the targets and their description. Figure $\mathbf{1}$ provides the structure of the secondary metabolites and plant sources. Tables 1-4 give Gold Fitness score and hydrogen bonding interaction values between all targets and secondary metabolites, interaction type, and bond length of the docking. The 3D and 2D interactions of the high scored metabolites with the target enzymes are shown in Figures (2-7). 


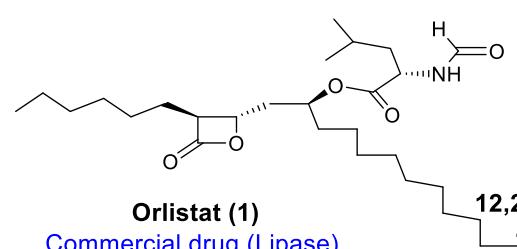

Commercial drug (Lipase) Source: Streptomyces toxytricini

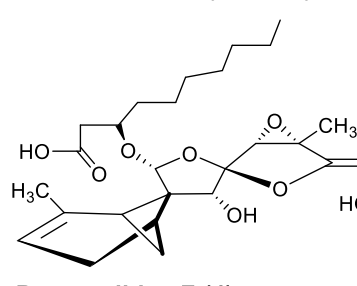

Purpurolides F (4) Citrus sinensis

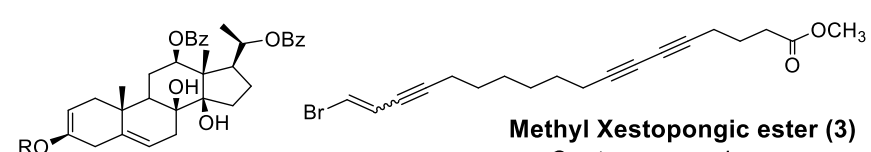

Centrosema pubescens
12,20-di-O-benzoyl-3ß,8ß,12ß,14ß,20-pentahydro
-(20R)-pregn-5-ene-3-O-ß-D-glucopyranosyl-

$(1 \rightarrow 4)$ B-D-digitaloside (2)

Dalbergia cochinchinensis

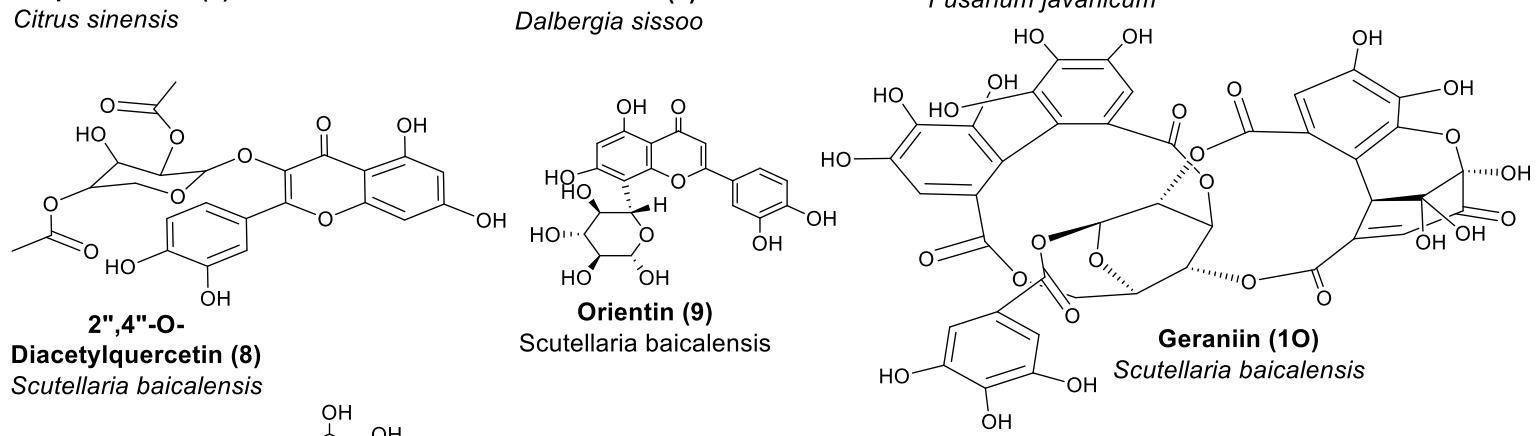

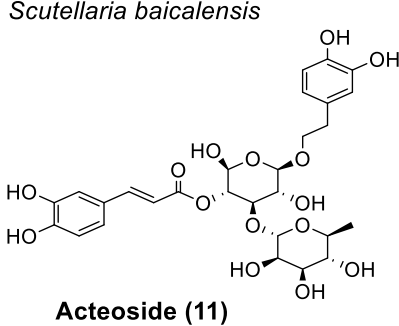

Acteoside (11)

Camelia sinensis<smiles>CC1OC(=c2c(=C3C=C=C(O)C=C3)oc3cc(O)cc(O)c3c2=O)C(OC2OC(CO)C(O)C(O)C2O)C(C(O)C(O)C(O)C(C)O)O1</smiles>

Kaempferol-3-O-

neohesperidoside(15)

Trifolium pratense

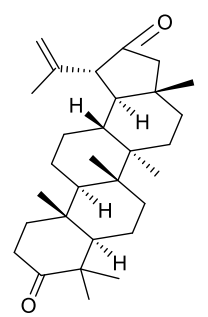

lup-20(29)-en-3,21-dione (19)

Salacia hainanensis

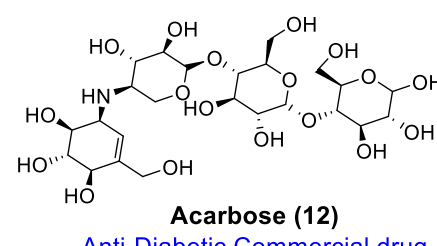

Anti-Diabetic Commercial drug Citrus depressa

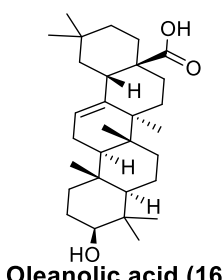

Oleanolic acid (16)

Manchurian apricot

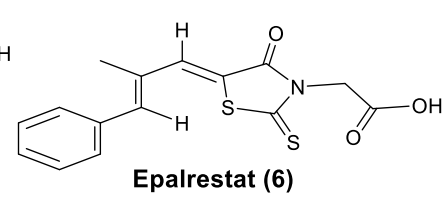

Commercial drug (Aldose reductase)

Fusarium javanicum

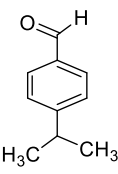

Cuminaldehyde (7)

Cuminum cyminum

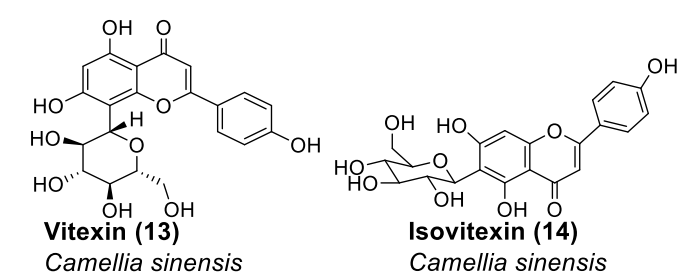

Camellia sinensis

Camellia sinensis

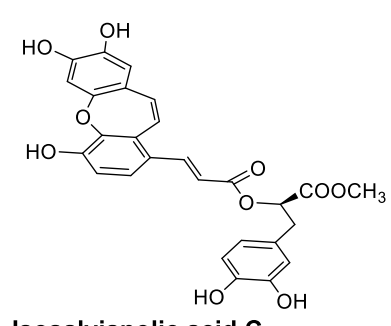

Isosalvianolic acid C methyl ester (18) Citrus paradisi

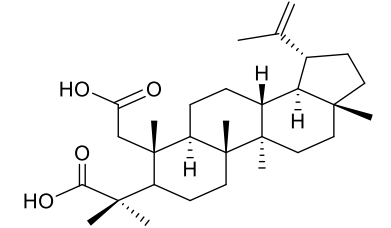

2,3-seco-lup-20(29)en-2,3-dioic acid (20) Citrus paradisi

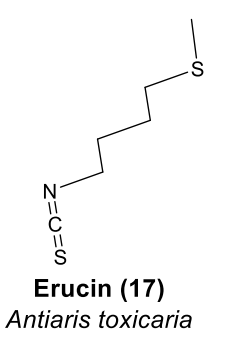

(1) 


\subsection{Docking results of pancreatic lipase with secondary metabolites}

In Table 1, methyl xestospongic ester (3) showed the best Gold fitness score of 65.83 and exhibited no any interaction with residues, but protein-ligand Vander Waals score is highest (vdw_ext $=49.52$ ) which is the focus part in this analysis, and pupurolide F (4) has a fitness score of 53.90 having three hydrogen bonds with the receptor (Asp79, Arg 256, Ala259). Binding modes of methyl xestospongic ester (3) and commercial drug, orlistat (1) in 2D and 3D interaction, are visualized in Figures 2-3, respectively. Among all, methyl xestospongic ester (3) has a better Gold Fitness Score when compare to orlistat (1) (Gold Fitness score is 64.49), a standard drug currently in the market. Likewise, purpurolide F (4), and schaftoside (5) have scores near to orlistat (1), and modifications may result in better ligands.

Table 1. Gold Fitness score and Protein-Ligand Interactions of Protein ID: 1LPB, Human Pancreatic Lipase Inhibition. The Gold Fitness score, interacting residues, type of interaction, bond length between residues, and ligands are shown.

\begin{tabular}{|c|c|c|c|c|}
\hline Title & Gold Score & Interactions & Type & Bond length $(\AA)$ \\
\hline \multirow{2}{*}{ 1. orlistat (commercial drug) } & \multirow{2}{*}{64.49} & Phe 77 & H-bond & 1.6 \\
\hline & & Ser 152 & H-bond & 2.7 \\
\hline \multirow{3}{*}{$\begin{array}{l}\text { 2.12,20-di-O-benzoyl-3 } 3,8 \beta, 12 \beta, 14 \beta, 20 \text {-pentah } \\
\text { ydroxy-(20R)-pregn-5-ene-3-O- } \beta \text {-D-glucopyra } \\
\text { nosyl-( }(1 \rightarrow 4) \beta \text {-D-digitaloside }\end{array}$} & \multirow[t]{3}{*}{18.8} & Phe 77 & H-bond & \\
\hline & & Asp 79 & H-bond & 3.4 \\
\hline & & $\operatorname{Trp} 252$ & $\mathrm{Pi}-\mathrm{Pi}$ & 1.8 \\
\hline \multirow[t]{2}{*}{ 3. methyl xestospongic ester } & \multirow[t]{2}{*}{65.83} & - & - & - \\
\hline & & Asp 79 & H-bond & 2 \\
\hline \multirow[t]{2}{*}{ 4. pupurolides $\mathrm{F}$} & \multirow[t]{2}{*}{53.9} & Arg 256 & H-bond & 2.2 \\
\hline & & Ala 259 & H-bond & 3.5 \\
\hline \multirow{2}{*}{ 5. schaftoside } & \multirow{2}{*}{50.14} & Phe 77 & H-bond & 1.6 \\
\hline & & His 263 & H-bond & 2.2 \\
\hline
\end{tabular}

Compound(12,20-di-O-benzoyl-3 $\beta, 8 \beta, 12 \beta, 14 \beta, 20$-pentahydroxy-(20R)-pregn-5-ene-3-O- $\beta$-D-glucop yranosyl- $(1 \rightarrow 4) \beta$-D-digitaloside) has the least score of 18.08 interacting with residues Phe77, Asp79, $\operatorname{Trp} 252$ in the active site of pancreatic lipase. Results suggested that methyl xestopongic ester (3) could be useful as a pancreatic lipase inhibitor as compared to orlistat, which is per IC 50 value 3.11 $\mu \mathrm{M}$ obtained from [15] (Table 2S). 


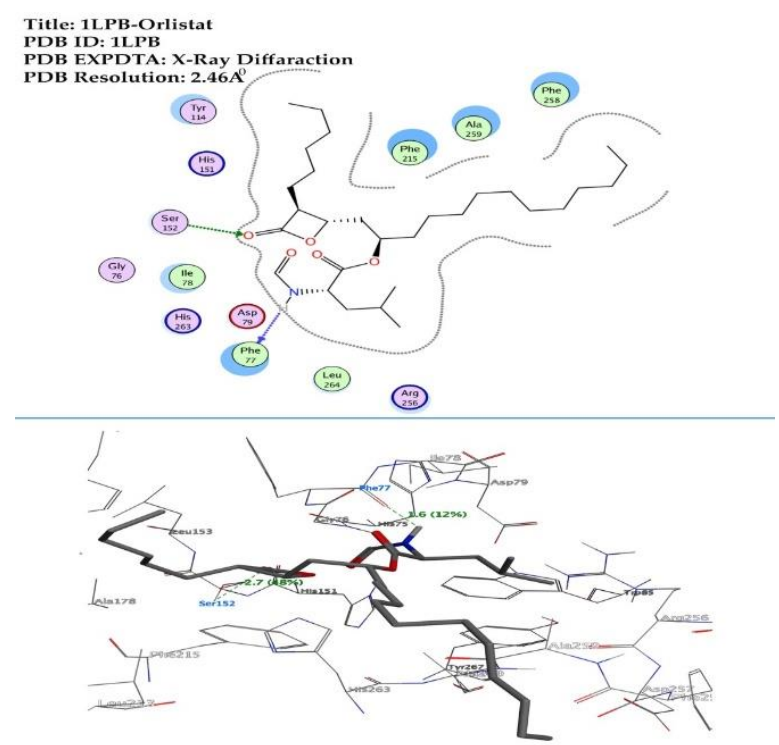

Figure 2. 2D (upper) and 3D (lower) interactions of pancreatic lipase (PDB ID: 1LPB) with orlistat (Fitness score of 64.49).

Title: 1LPB-Methy xestopongic ester

PDB ID: 1LPB

PDB EXPDTA: X-Ray Diffaraction

PDB Resolution: 2.46A
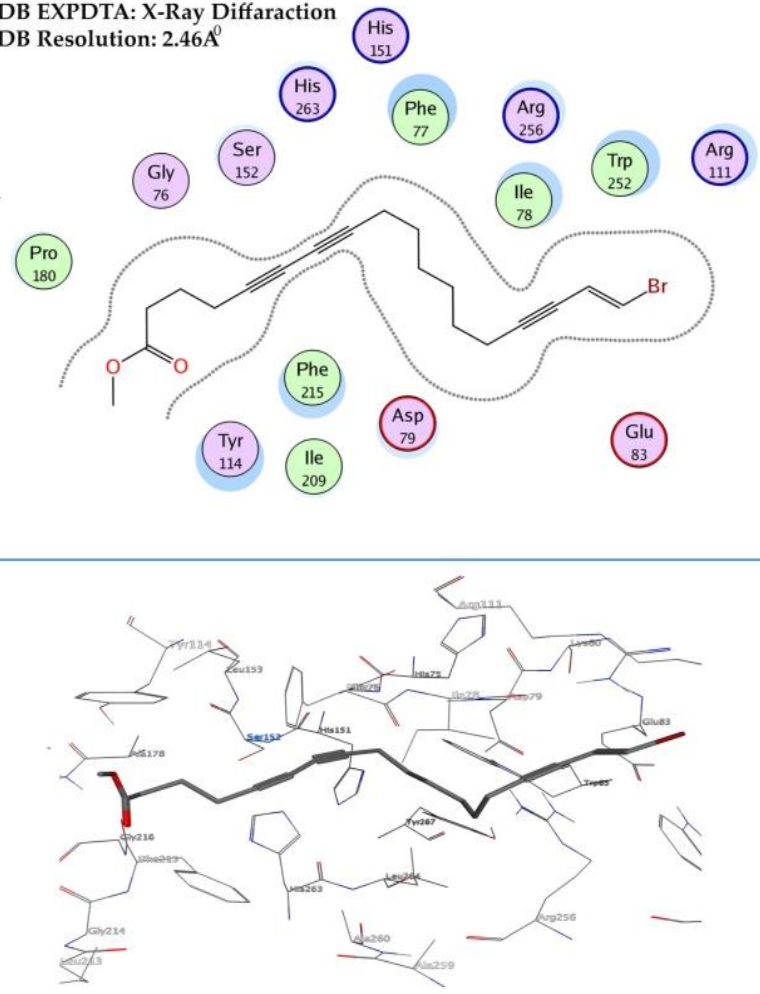

Figure 3. 2D (upper) and 3D (lower) interactions of pancreatic lipase (PDB ID: 1LPB) with methyl xestospongic ester (Fitness score of 65.83).

2.2 Docking results of Aldose Reductase with secondary metabolites 
Table 2 reveals the metabolites as aldose reductase inhibitor, the interacting amino acid residues, the Gold Score, the number of the hydrogen bonds. The commercial drug, epalrestat (6), is utilized as a positive control, and its interaction with the enzyme is visualized in Figure 4. Docking of

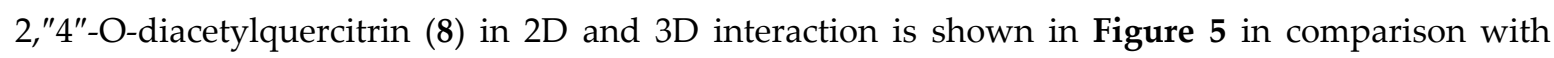
epalrestat (6). The putative binding modes of metabolites inside the active site pocket of aldose reductase are shown in Table 2 . Inside the active pocket of aldose reductase, $2, " 4$ "-O-diacetylquercitrin (8) was determined to be fitted with highest nearby Gold Fitness score of 65.15 with amino acid residue interaction of Tyr 48, Trp111, Ser 159, and Tyr 209 while the Fitness score of epalrestat (6), a commercial drug, inside the active pocket was found to be 66.76 which showed interaction with Tyr 209, Lys 262. Geraniin (10) and acteoside (11) have nearby Gold Fitness scored of 51.87 and 58.65, respectively. The result suggested that 2,"4"-O-diacetylquercitrin (8) could be useful as an aldose reductase inhibitor which also shows a significant IC50 value of 0.077 $\mu \mathrm{M}[16]$ (Table 3S).

Table 2. Gold Fitness score and Protein-Ligand Interactions of Protein ID: 1USO aldose reductase inhibition. The Gold Fitness score, interacting residues, type of interaction, bond length between residues, and ligands are shown.

\begin{tabular}{lcccc}
\hline Title & Gold Score & Interactions & Type & $\begin{array}{c}\text { Bond } \\
\text { length (Å) }\end{array}$ \\
\hline & & Tyr 209 & Pi-Pi & - \\
6. epalrestat (commercial drug) & 66.76 & Lys 262 & H-bond & 1.80 \\
& & Ser 263 & H-bond & 2.40 \\
7. cuminaldehyde & & Asn 160 & H-bond & 1.60 \\
& 42.05 & Tyr 209 & H-bond & 2.20 \\
8. 2,"4"-O-diacetylquercitrin & & Tyr 48 & H-bond & 2.00 \\
& & Trp 111 & Pi-Pi & - \\
9. orientin & \multirow{2}{*}{65.15} & Ser 159 & H-bond & 2.30 \\
& & Tyr 209 & Pi-Pi & - \\
10. geraniin & & Lys 262 & H-bond & 1.7 \\
& & Val 264 & H-bond & 1.9 \\
& 52.27 & Ser 305 & H-bond & 1.1 \\
& & Lys 307 & H-bond & 2 \\
11. acteoside & 51.87 & Val 47 & H-bond & 1.9 \\
& & His 100 & H-bond & 2.8
\end{tabular}


Title: 1USO-Epalrestat

PDB ID: 1USO

PDB EXPDTA: X-Ray Diffaraction

PDB Resolution: 0.66

(GIm)

Pro

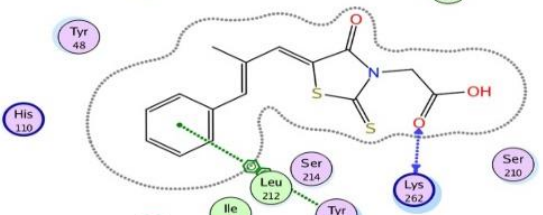

(AT2) (160)

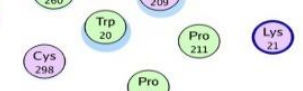

(P15)

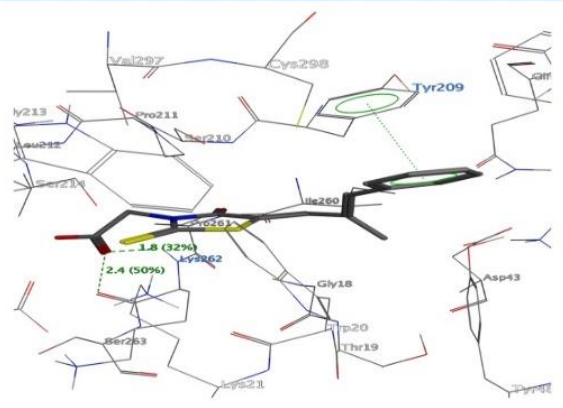

Fig. 4. 2D (upper) and 3D (lower) interactions of aldose reductase (PDB ID: 1US0) with epalrestat (Fitness score of 66.76).

Title: 1US0- 2, "4" - O-diacetylquercetrin

PDB ID: 1USO

X-Ray Diffaraction

PDB Resolution: $0.66 A^{\circ}$
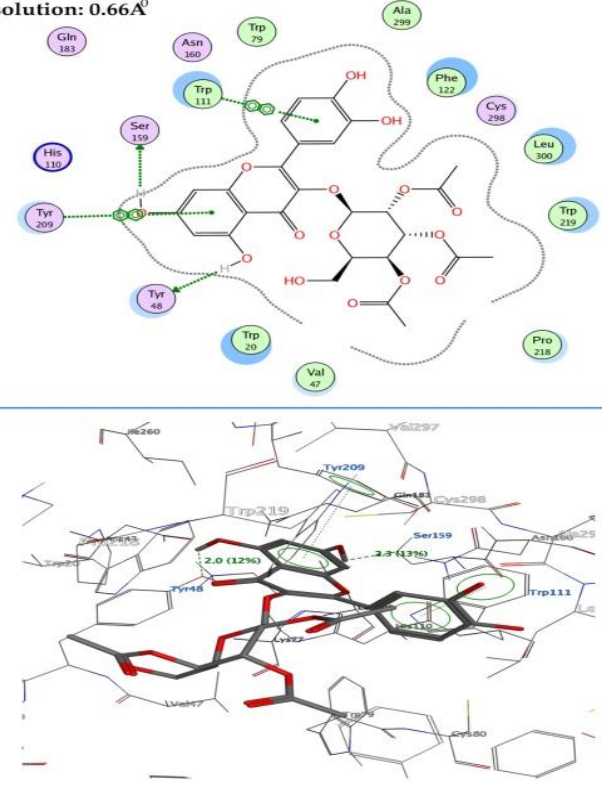

Fig. 5. 2D (upper) and 3D (lower) interactions of aldose reductase (PDB ID: 1US0) with 2,"4"-O-diacetylquercitrin (Fitness score of 65.15). 


\subsection{Docking results of alpha-amylase with secondary metabolites}

Molecular docking studies of metabolites revealed their interaction modes within the active site of $\alpha$ -amylase. The different Fitness scores calculated for all the metabolites were shown in Table 3. In the docking studies of the metabolites with $\alpha$-amylase, kaempferol-3-O-neohesperidoside (15) exhibited the highest Fitness score of 53.37, whereas the reported $\mathrm{IC}_{50}$ value was $0.080 \mu \mathrm{M}$ (Table $4 \mathrm{~S}$ ). The key interacting amino acid residues of the enzymes with the top-scored kaempferol-3-O-neohesperidoside (15) and acarbose (12) (standard drug) were Try 61, Gln 62, His 100, Asp 196, Glu 232, Arg 194, Asp 299, Asp 355 as shown in Figures 6.

Table 3. Gold Fitness score and Protein-Ligand Interactions of Protein ID: 5U3A pancreatic $\alpha$-amylase inhibition. The Gold Fitness score, interacting residues, type of interaction, bond length between residues, and ligands are shown.

\begin{tabular}{|c|c|c|c|c|}
\hline Title & $\begin{array}{l}\text { Gold } \\
\text { Score }\end{array}$ & Interactions & Type & $\begin{array}{l}\text { Bond length } \\
\text { (Å) }\end{array}$ \\
\hline \multirow[t]{5}{*}{ 12. acarbose (commercial drug) } & 34.52 & His 100 & $\mathrm{H}$ - bond & 2.6 \\
\hline & & Asp 196 & H-Bond & 2.1 \\
\hline & & His 298 & H-Bond & 3.5 \\
\hline & & Asp 299 & 3 H-Bonds & $1.6,1.9,2.4$ \\
\hline & & Asp 355 & H-bond & 2.8 \\
\hline \multirow[t]{4}{*}{ 13. vitexin } & 49.79 & Gln 62 & H-Bond & 2.2 \\
\hline & & Tyr 150 & H-Bond & 2.9 \\
\hline & & Asp196 & H-bond & 1.7 \\
\hline & & Glu232 & H-bond & 1.9 \\
\hline \multirow[t]{2}{*}{ 14. isovitexin } & 45.65 & Asp 196 & Pi-Pi & 1.9 \\
\hline & & Tyr 150 & $\mathrm{Pi}-\mathrm{Pi}$ & - \\
\hline \multirow[t]{7}{*}{ 15.kaempferol-3-O-neohesperidoside } & 53.37 & Tyr 61 & H-Bond & 2.3 \\
\hline & & Gln 62 & H-bond & 2 \\
\hline & & Asp 196 & H-Bond & 2 \\
\hline & & Glu 232 & H-Bond & 3.4 \\
\hline & & Arg 194 & H-Bond & 2.2 \\
\hline & & Asp 299 & H-Bond & 2.6 \\
\hline & & Asp 355 & H-Bond & 1.7 \\
\hline \multirow[t]{2}{*}{ 16. oleanolic acid } & 20.27 & Glu 232 & 2H-Bond & $1.80,2.40$ \\
\hline & & His 298 & - & 2.8 \\
\hline 17. erucin & 41.38 & - & - & - \\
\hline
\end{tabular}




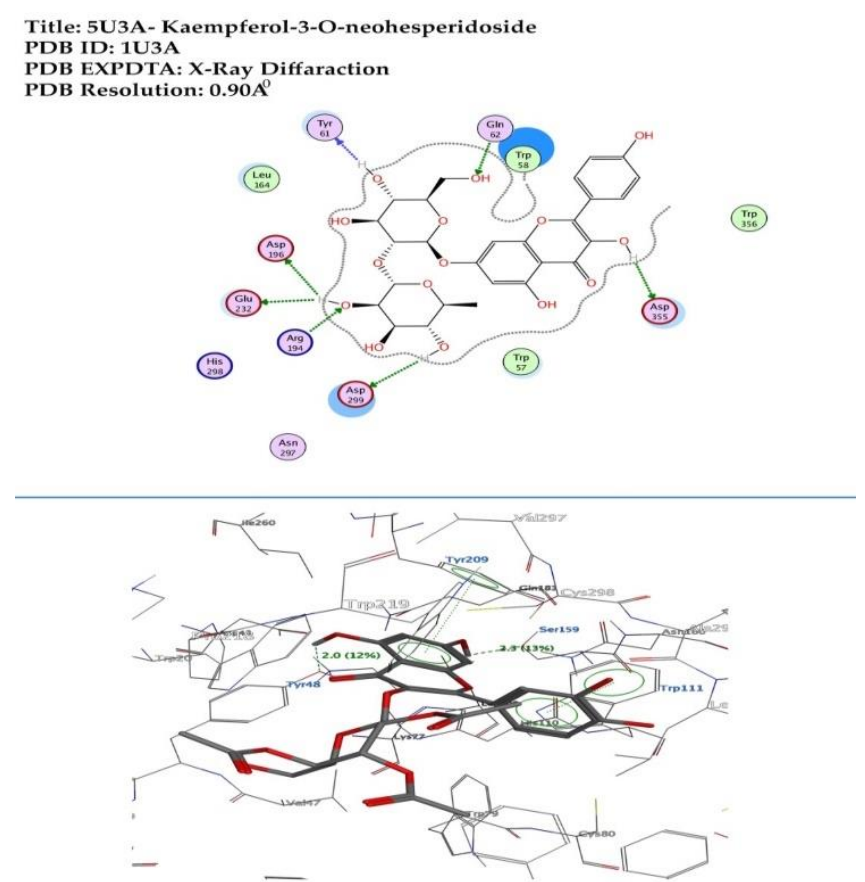

Fig. 6. 2D (upper) and 3D (lower) interactions of $\alpha$-amylase (PDB ID: 5U3A) with kaempferol-3-O-neohesperidoside (Fitness score of 53.37).

\subsection{Docking analysis of alpha-glucosidase with secondary metabolites}

Molecular docking with $\alpha$-glucosidase using active metabolites (Table 5S) revealed the different interaction modes and binding interaction in the active pocket of the enzyme. The trend was different than observed for the commercial drug, acarbose (12). The Gold Fitness score for the molecular docking studies of the metabolites was found to be in the range of 11.68 to 53.44 . The Fitness score value of isosalvianolic acid C methyl ester (18) is 53.44, which was highest among others. The result from the docking analysis also showed that N-trans-Caffeoyl-tyramin (21) has a nearby fitness score of 50.59 and 2,3-seco-lup-20(29)en-2,3-dioic acid (20) has the least score in the series. Amino acid residues modes of interaction within the active pocket of the $\alpha$-glucosidase with the metabolites were presented in Figures 7. Ileu 18, Trp 179, and Arg 181 were involved in active sites and interacted with top-scored isosalvianolic acid $\mathrm{C}$ methyl ester. 
Table 4. Gold Fitness score and Protein-Ligand Interactions of Protein ID: 5NN8 $\alpha$-glucosidase inhibition. The Gold Fitness score, interacting residues, type of interaction, bond length between residues, and ligands are shown.

\begin{tabular}{|c|c|c|c|c|}
\hline Title & Gold Score & Interactions & Type & $\begin{array}{l}\text { Bond } \\
\text { length (A) }\end{array}$ \\
\hline \multirow[t]{6}{*}{ 1. acarbose (commercial drug) } & 34.88 & Ileu 18 & H-bond & 3.5 \\
\hline & & Asp11 & H-bond & 3.1 \\
\hline & & $\operatorname{Trp} 39$ & Pi-cation & - \\
\hline & & Arg 181 & H-bond & 2.1 \\
\hline & & Tyr 449 & H-bond & 3.4 \\
\hline & & Val 450 & H-bond & 1.6 \\
\hline \multirow[t]{3}{*}{ 18. isosalvianolic acid $C$ methyl ester } & 53.44 & Ileu 18 & H-bond & 1.8 \\
\hline & & $\operatorname{Trp} 179$ & H-bond & 1.8 \\
\hline & & Arg 181 & H-bond & 1.5 \\
\hline 19. lup-20(29)-en-3,21-dione & 12.88 & - & - & - \\
\hline \multirow[t]{2}{*}{ 20. 2,3-seco-lup-20(29)en-2,3-dioic acid } & 11.68 & Pro 14 & H-bond & 3 \\
\hline & & Arg 237 & $2 \mathrm{H}$-bond & $2.40,1.70$ \\
\hline \multirow[t]{3}{*}{ 21. N-trans-Caffeoyl-tyramin } & 50.59 & Asp 11, & H-bond & 1.9 \\
\hline & & Arg 181 & H-bond & 1.9 \\
\hline & & Val 450 & H-bond & 3.1 \\
\hline
\end{tabular}

Title: 5NN8- Isosalvianolic C methyl ester

PDB ID: 5NN8

X-Ray Diffaraction

PDB Resolution: $2.45 \mathrm{~A}^{\circ}$

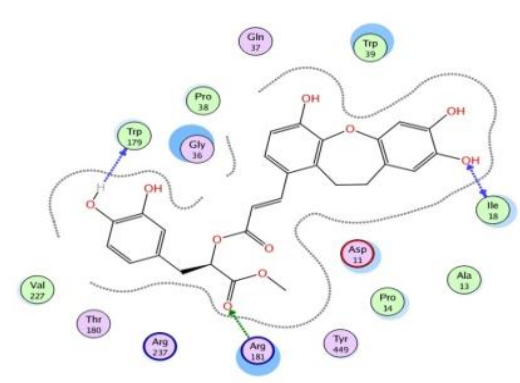

(ii)

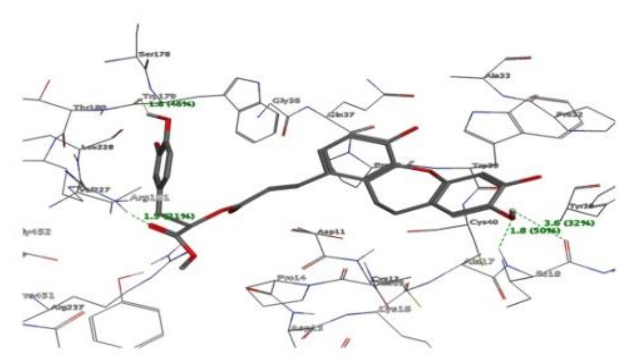

Fig.7. 2D and 3D interactions of alpha-glucosidase (PDB ID: 5NN8) with isosalvianolic acid C methyl ester (Fitness score of 53.44). 


\subsection{Toxicity analysis by Pro Tox-II}

The secondary metabolites expecting to inhibit different enzymes were studied by Pro Tox-II. However, some compounds like (-) gallocatechin (non-toxic), erucin (non-toxic), pectin (non-toxic), daucosterol (immunotoxicity with probability 0.99 ) were classified under class 6 as shown in Table 6S. The class toxicity of the secondary metabolites ranges from 6-2, whereas sinigrin isolated from brassiaciae plant was non-toxic under toxicity class 2 . Nevertheless, for the convenient study, only potent secondary metabolites were taken based on $\mathrm{IC}_{50}$ values for in-silico analysis. Based on the analysis, secondary metabolites having the best Gold Score were found to be classified under class 5 except methyl xestospongic ester (non-toxic), which was categorized under class 4 . The confidence score determines the toxicity prediction level based on the target. Additionally, about 29 secondary metabolites were non-toxic. All the secondary metabolites were found to have high toxicity with a confidence score of more than 0.7 . Secondary metabolites were found to have high toxicity with a confidence score of more than 0.7. Metabolites such as 2,"4"-O-diacetylquercitrin, .kaempferol-3-O-neohesperidoside, isosalvianolic acid C methyl ester were found to have immunotoxicity with a confidence score of more than 0.7. All the metabolites were found to be inert towards other toxicological targets, as illustrated in Table 6S. From the above in silico toxicity prediction experiment, it was inferred that orlistat was found to be within the range of confidence score 0.7 , while other secondary metabolites showed potential for having immunotoxicity. Though the software was operated based on a limited number of databases, this toxicity prediction may help to decode the possible molecular mechanism, which leads to toxicity. The vast number of databases and assessing parameters must be added for the complete toxicity prediction. Some other toxicity parameters like genotoxicity, nephrotoxicity, and cardiotoxicity must be added to gain knowledge on toxicity prediction of the metabolites

\subsection{Enzymes of Diabetic Target}

$\alpha$-Amylase (EC 3.2.1.1 and P04745) can be found in microbes, plants, and animals (Anitha Gopal \& Muralikrishna, 2009). It is found in saliva and pancreatic juice [18] that hydrolyses $\alpha$-linked bond in any substrate resulting in glucose and maltose that could quickly enter the bloodstream [8]. Inhibition of $\alpha$-amylase, in turn, decreases the digestion of starch in the intestine, which helps to avoid hyperglycemia and maintain normal blood sugar levels [19]. In the present context, antidiabetic drugs used for the management of glucose come with some side effects. Therefore, great concern is given to developing the therapeutics for diabetes and potent inhibitors against this enzyme from secondary metabolites [20], [21]. 6-Gingerol and oleanolic acid isolated from Aframomum melegueta K. Schum showed good inhibitory activity against $\alpha$-amylase with $\mathrm{IC}_{50}$ $81.78 \mu \mathrm{M}$ and $91.72 \mu \mathrm{M}$, respectively in the non-competitive mode of action [22].

Likewise, gedunin and azadiradione isolated from Azadirachta indica exhibited potential inhibition with an IC 50 value of 74.17 and $68.38 \mu \mathrm{M}$, respectively. Cytotoxicity and bioactivity of azadiradione (3.5-17.7 $\mu \mathrm{M})$, gedunin (3.3-16.5 $\mu \mathrm{M})$ on the $\alpha$-secretory cell line AR42J were found to be cytotoxic (IC50 values of 11.1, and 13.4 respectively [23]. Sinigrin of Brassicaceae family binds competitively in the active site of $\alpha$-amylase with $\mathrm{IC}_{50}$ value $0.00124 \mu \mathrm{M}$ as compared to standard acarbose (IC50 value of $0.0017 \mu \mathrm{M}$ ) when administered at $150 \mathrm{mg} / \mathrm{kg}$ to STZ induced zebrafishes [24]. Betulin 
isolated from Ruellia tuberosa L. strongly inhibited $\alpha$-amylase with IC 50 value $0.14 \pm 0.005 \mathrm{mg} / \mathrm{mL}$ in the non-competitive mode of action (Ki: $314 \mu \mathrm{M})$, which significantly reduces blood glucose upon administered at $450 \mathrm{mg} / \mathrm{kg}$ single dose for 14 days in low dose streptozotocin (MLD-STZ) induced rats [25]. Other results are mentioned in Table 4S.

$\alpha$-glucosidase (EC 3.2.1.20 and P10253) is a membrane-bound enzyme located at the epithelium of the small intestine, which hydrolyzed the cleavage of polysaccharides and releases glucose (Standl\& Schnell, 2012). Some commercially available $\alpha$-glucosidase inhibitors usually cause adverse effects. Noticeably, epicatechin-(43,8)-epicatechin gallate (B2-3'-O-gallate) isolated from roots of Rhodiola crenulata inhibit $\alpha$-glucosidase with $\mathrm{IC}_{50}$ values of $(0.31 \pm 0.01) \mu \mathrm{M}$ and shows mixed-competitive inhibition character $(\mathrm{Ki} \quad 0.30 \pm 0.03 \mu \mathrm{M})$. Similarly, 2-(4-hydroxyphenyl) ethyl 3,4,5-trihydroxybenzoate was a competitive inhibitor with $\mathrm{Ki}$ value of $(3.10 \pm 0.09) \mu \mathrm{M}$ [26].1,7-bis(4-hydroxyphenyl)heptane-3,5-diol isolated from the tuberous rhizomes of Dioscorea oppositaThunb inhibit $\alpha$-glucosidase significantly with IC 50 values $0.38 \mathrm{mM}$ [27]. Papyriflavonol, A isolated from roots of Broussonetia papyrifera, showed $\alpha$-glucosidase inhibition with IC $_{50}$ values of $2.1 \pm 0.2 \mu \mathrm{M}$. Kinetics studies showed that it shows mixed-type inhibition with Ki values of $2.3 \pm 0.3$ $\mu \mathrm{M}[28]$.

Several meroterpenoids were isolated from Ganoderma leucocontextum. Among them ganomycin I displayed the most potent inhibitor of $\alpha$-glucosidase from Baker's yeast and rat with IC 50 value 0.3 \pm 0.1 and $0.4 \pm 0.1 \mu \mathrm{M}$, respectively. The kinetic study revealed a non-competitive type of inhibition and did not show any toxic effect during $24 \mathrm{~h}$. After 3 weeks of treatment with inhibitor compounds significantly decreases blood glucose levels by oral sucrose tolerance test (OSTT) in KK-Ay mice [29]. Other results are mentioned in Table 5S.

Aldose Reductase (EC 1.1.1.21 and P15121) is the rate-limiting in the polyol pathway, which reduces glucose to sorbitol, which is further converted into fructose by fructose dehydrogenase enzyme. The enzyme consists of 315 amino acids and has a parallel $\beta$ 8/ $\alpha 8$ barrel structure (Wilson et al., 1992). It has three distinct binding pockets: 1) 'anion binding pocket,' made up of Tyr 48 , His 110, Trp 20, Trp 111 and positively charged NADP+; 2) 'hydrophobic pocket' or `specificity pocket' lined by the amino acid residues Leu 300, Cys298, Cys 303, Trp 111 and Phe 122 and 3) 'hydrophobic pocket' constituted by the amino acid residues Trp20, Trp111, Phe122, and Trp219 (Zhu, 2013). The reaction consists of two steps; first is the transfer of hydride from the NADPH to the carbonyl substrate, and the second is the donation of proton for the reduction of carbonyl to alcohol either by Tyr 48 or His110 (Blakeley et al., 2008). Biochemical and crystallographic studies have suggested that ARIs preferably bind to the ALR2/NADP complex, whereas the substrates bind to the ALR2/NADPH form (Ehrig et al., 1994).

Studies have been carried out which shows that the effects drugs have hydrophilic and hydrophobic groups (one or two aromatic groups); hydrophilic groups bind to the His110 or Tyr 48 of the anion binding pocket which hinders the proton transfer to the carbonyl group whereas the hydrophobic groups bind to the specific pocket. Moreover, inhibitors specific to ALR2 interact with C-terminal residues that are not conserved in the homologous aldehyde reductase (ALR1) [30]. 
Coumarin groups have been found to inhibit the enzyme competitively, non-competitively, and uncompetitively [31]. It has been found that the flavonoid rutin inhibits the enzyme in an uncompetitive manner $\left(\mathrm{Ki}=25^{*} 10^{-6} \mathrm{M}\right)$, and the inhibition is found to be more specific towards ALR2 compared to ALR1 [32]. With the increase in the concentration of the curcumin and ellagic acid Vmax decreases but the $\mathrm{Km}$ remains constant indicating the non-competitive mode of inhibition; the enzyme binds to the enzyme nucleotide complex than the free enzyme [33], [34]

Various researches have been conducted to find the effectiveness of natural products for the inhibition of Aldose Reductase. Phenolic (gallotannin) compounds also have aldose reductase inhibiting properties; pistafolin B from Caesalpinia spinose binds to the active site of aldose reductase the $\mathrm{IC}_{50}$ value is $0.198 \mathrm{mM}$ and $\mathrm{Ki}$ value is $0.34 \mathrm{mM}$ the compound has found to show mixed-type inhibition [35]. Trans cinnamaldehyde from the bark of Cinnamomum cassia is also effective for inhibiting rat lens Aldose Reductase ( $\mathrm{IC}_{50} 0.003 \mathrm{mg} / \mathrm{ml}$ ) though it is less as compared to the control $\begin{array}{lllll}\text { quercitrin } & \left(\mathrm{IC}_{50}\right. & 0.0005 & \mathrm{mg} / \mathrm{ml}) & \text { [36]. }\end{array}$ 3-O-a-L-rhamnopyranosyl-(1.6)--B-D-glucopyranoside and isorhamnetin 3-O--a-L-rhamnopyranosyl-(1.6)--B-D-glucopyranoside, were determined to exhibit the highest degree of rat lens aldose reductase inhibitory activity in vitro, evidencing IC $_{50}$ values (concentration required for a $50 \%$ inhibition of enzyme activity) of 5.6 and $9.0 \mu \mathrm{M}$, respectively [37]. Other results are mentioned in Table 3S.

Lipase (triacylglycerol acylhydrolase, EC 3.1.1.3, and P16233) is responsible for the absorption of dietary triglycerides, so its inhibition declines fat absorption [38] and it could be useful for the treatment of obesity (Carrière et al., 1997; Lengsfeld et al., 2005). Orlistat is only commercially available pancreatic lipase inhibitor, but it causes adverse gastrointestinal reactions such as oily stools, oily spotting, and flatulence. Quercetin (IC50: $53.05 \mu \mathrm{M}$ ), kaempferol (IC50: $79.38 \mu \mathrm{M}$ ), myricitrin (IC50: $92.85 \mu \mathrm{M})$, quercitrin (100.56), and avicularin $(141.84 \mu \mathrm{M})$ show inhibition against this enzyme. Myricitrin, quercitrin, and avicularin, which belong to flavonol-3-O-glycosides showed non-competitive or mixed-type inhibition, whereas flavonol aglycones (quercetin and kaempferol) showed competitive inhibition (Park et al., 2019).

Purpurolide F showed significant inhibitory activity against pancreatic lipase (PL) with an IC 50 value of $1.22 \mu \mathrm{mol} / \mathrm{L}$ kaempferol $\left(\mathrm{IC}_{50}=1.50 \mu \mathrm{mol} / \mathrm{L}\right)$. Cytotoxicity of compounds was tested on human cancer cell lines, including HCT-116 colon, BGC-823 stomach, and Bel-7402 hepatoma cell lines and found to be non-toxic at $50 \mu \mathrm{mol} / \mathrm{L}$. So it might be viable candidates for further clinical development as PL inhibitor (Xia et al., 2020). Other results are mentioned in Table 2S.

\section{Discussion}

The major metabolites, methylxestospongic ester, schaftoside, and purpurolide $\mathrm{F}$ isolated from Xestospongia testudinariamay., Trigonella foenum-graecum (seeds), and Penicillium purpurogenum IMM 003 respectively with Gold score 65.83, 50.14, 53.9 followed by IC $503.11 \mu \mathrm{M} .230 .29 \mu \mathrm{M}, 1.22 \mu \mathrm{M}$, respectively, could be the possible potent drug for lipase as there was no toxicity effect based on Pro Tox II software [15] [40] [41]. The aldose reductase inhibitors 2,"4"-O-diacetylquercitrin , acteoside, 
cuminaldehyde, orientin, and geraniin with Gold score 65.15, 58.65, 42.0552 .27 , and 51.87 could be a potential drug for aldose reductase because of possessing the high binding capacity of protein.

Likewise, kaempferol-3-O-neohesperidoside isolated from Solenostemma argel with Gold score 53.37 and IC $500.08 \mu \mathrm{M}$ could be a potential drug for $\alpha$-amylase [42]. Moreover, vitexin, isovitexin and oleanolic acid follows order of Gold score and IC 50 values 49.79, $0.046 \mu \mathrm{M}>45.65,0.138>20.27,0.219$ respectively could be potential drug of $\alpha$-amylase [43] [44]. Additionally, erucin isolated from Eruca vesicaria with Gold score 41.38 followed by IC $_{50} 0.315$ could be possible inhibitor candidate for $\alpha$-amylase inhibition as it didn't show any toxicity effect [45]. Similarly, for $\alpha$-glucosidase isosalvianolic acid C methyl ester isolated from Salvia miltiorrhiza Bge and N-trans-Caffeoyl-tyramin from Polygonum hyrcanicum possessing highest Gold score values 53.44 and 50.59 respectively than acarbose (Gold Score 34.88) could be used for $\alpha$-glucosidase inhibition [46] [47].

The molecular docking of 18-secondary metabolites isolated from the various plants along with standard drugs like orlistat (lipase), epalrestat (aldose reductase), acarbose ( $\alpha$-amylase and $\alpha$-glucosidase) docked by using Gold software and processed by using MOE software to predict the binding modes of these drug-like compounds. The results showed that methyl xestospongic ester with Gold Score 65.83 and IC50 $3.11 \mu \mathrm{M}, 2$,"4"-O-diacetylquercitrin (Gold Score 65.15, IC50 $0.077 \mu \mathrm{M}$ ), kaempferol-3-O-neohesperidoside (Gold Score 53.37, IC $50.08 \mu \mathrm{M}$ ) and isosalvianolic acid C methyl ester (Gold Score 53.44, IC $50111.9 \times 10^{-3} \mu \mathrm{M}$ ) possessed striking interactions with active site residues of the target protein for lipase, aldose reductase, $\alpha$-amylase, and $\alpha$-glucosidase respectively. The in silico toxicity of the metabolites was expected by using ProTox-II software which revealed that orlistat was within the range of confidence score 0.7 having hepatoxicity, while best secondary metabolites 3 (Table 1), 8 (Table 2), 15 (Table 3), and 19 (Table 4), have shown immunotoxicity. Although few of the metabolites were reported in the in-vitro study earlier, the novelty of this investigation can be justified only by complete analysis. Moreover, in silico toxicity of the metabolites were also not known to date. However, the exact toxicity profile of secondary metabolites with the best Gold Score should only be concluded after detailed real-time in-vitro, ex vivo, and in vivo toxicity studies.

Taken together, the current study provides a computational background for lipase, aldose reductase, $\alpha$-amylase, and $\alpha$-glucosidase inhibitors. Future studies should more carefully examine the clinical efficacy of these compounds, thus facilitating the development of new resources for the treatment of diabetes mellitus.

\section{Materials and Methods}

\subsection{Protein selection and preparation}

The crystal structures of the selected proteins were retrieved from the protein data bank (PDB database, www.rcsb.org). The PDB numbers of each protein were 1LPB (Human Pancreatic Lipase), 1US0 (Aldose reductase), 5U3A (Pancreatic $\alpha$-amylase), 5NN8 ( $\alpha$-glucosidase). The downloaded protein structure was prepared before docking using MOE 2009. Protein preparation was done by preprocessing the structures by assignment of bonds and bond orders, the addition of hydrogen's, filling in missing loops or side chains, capping uncapped $\mathrm{C}$ and $\mathrm{N}$ termini, adjusting bonds and formal charges for metals, and correcting mislabeled elements, 
removing water molecules, removing unwanted chains and optimization of hydrogen-bonded structures followed by minimization.

\subsection{Ligand preparation and molecular docking}

The structures of the selected secondary metabolites were downloaded from Pubchem https://pubchem. ncbi.nlm.nih.gov/) and saved in mol format. The energy minimization was done using the Lig- $\mathrm{X}$ module. The minimized structures were docked on the prepared protein using GOLD. The best metabolites were identified based on the Higher Fitness score based on free energy and interaction with amino acid residues for each protein.

\subsection{Molecular docking protocol}

The molecular docking was achieved using GOLD version 4.0.1. to predict the scores for interactions of the targeted protein-ligand (Verdonck et al. 2003). GOLD uses a genetic algorithm for docking and performs automated docking with full ligand flexibility and partial flexibility in the neighborhood of the protein active site (Spassov et al. 2008). The stability of the docked ligand-protein complex is due to hydrogen bonding and Vander Waals interactions. The sites of binding of the residues of amino acids of the defined proteins have been determined from crystal structure and literature review. This was conducted for atoms with-in $10 \AA$ of the submitted binding residues in the pocket of binding and final docking was carried out for result analysis. Docking results of the selected target with commercial drugs and top-scored metabolites were tabulated in Table 1-4. The compounds with the highest fitness score were considered having good binding affinity according to Gold Score molecular mechanics function as,

$$
\text { Gold Fitness }=\text { Shb_ext }+1.375(\text { Svdw_ext })+\text { Shb_int }+ \text { S(vdw_int })
$$

Where Shb_ext is the protein-ligand hydrogen-bond score, and Svdw_ext is the protein-ligand Van der Waals score. Shb_int is the contribution to the fitness due to intramolecular hydrogen bonds in the ligand; this term is switched off in all calculations presented in this work: Svdw_int is the contribution due to intramolecular strain in the ligand. The compound, which is having the highest fitness score, is having the highest binding affinity.

\subsection{ProTox-II}

Protox-II incorporates molecular similarity, pharmacophores, fragment propensities, and machine learning models for the prediction of various toxicity endpoints, such as acute toxicity, hepatotoxicity, cytotoxicity, carcinogenicity, mutagenicity, immunotoxicity, adverse outcomes pathways, and toxicity targets. ProTox-II provides a freely available web server for in-silico toxicity prediction for toxicologists, regulatory agencies, computational and medicinal chemists, and all users without login at [39].

Supplementary Materials: Available 
Author Contributions: Babita Aryal, Bishnu P. Marasini, Karan Khadayat, Darbin Kumar Poudel, Ganesh Lamichhane, Prakriti Budhathoki, Purushottam Niraula, Sonika Dawadi, Rishab Marahatha, Sitaram Phuyal, and Niranjan Parajuli have contributed equally to review the literature on inhibition of metabolic enzymes. Saroj Basnet performed In-Silico analysis of targeted drug candidates. This project was coordinated by Niranjan Parajuli.

Funding: This research was funded by the Higher Education Reform Project (HERP), Tribhuvan University, Kathmandu, Nepal.

Abbreviations: NEFA: Non-esterified fatty acid; LDL-C: Low-density lipoprotein cholesterol; AR: Aldose reductase

Acknowledgments: This project is supported by the Higher Education Reform Project (HERP), Tribhuvan University.

Conflict of Interests: The authors declare that they have no known competing for financial interests or personal relationships that could have appeared to influence the work reported in this paper.

\section{REFERENCES}

[1] M. Ekor, "The growing use of herbal medicines: issues relating to adverse reactions and challenges in monitoring safety," Front. Pharmacol., vol. 4, 2014, doi: 10.3389/fphar.2013.00177.

[2] R. A. Halberstein, "Medicinal Plants: Historical and Cross-Cultural Usage Patterns," Ann. Epidemiol., vol. 15, no. 9, pp. 686-699, Oct. 2005, doi: 10.1016/j.annepidem.2005.02.004.

[3] D. J. Newman, "Natural Products as Leads to Potential Drugs: An Old Process or the New Hope for Drug Discovery?," J. Med. Chem., vol. 51, no. 9, pp. 2589-2599, May 2008, doi: 10.1021/jm0704090.

[4] J. D. Phillipson, "Phytochemistry and medicinal plants," Phytochemistry, vol. 56, no. 3, pp. 237243, Feb. 2001, doi: 10.1016/S0031-9422(00)00456-8.

[5] P. Saeedi et al., "Global and regional diabetes prevalence estimates for 2019 and projections for 2030 and 2045: Results from the International Diabetes Federation Diabetes Atlas, 9th edition," Diabetes Res. Clin. Pract., vol. 157, Nov. 2019, doi: 10.1016/j.diabres.2019.107843.

[6] R. Williams et al., IDF Atlas 9th Edition 2019. 2019.

[7] American Diabetes Association, "2. Classification and Diagnosis of Diabetes: Standards of Medical Care in Diabetes -2019," Diabetes Care, vol. 42, no. Supplement 1, pp. S13-S28, Jan. 2019, doi: 10.2337/dc19-S002.

[8] A. Chaudhury et al., "Clinical Review of Antidiabetic Drugs: Implications for Type 2 Diabetes Mellitus Management," Front. Endocrinol., vol. 8, Jan. 2017, doi: 10.3389/fendo.2017.00006.

[9] S.-H. Hsieh, K.-C. Shih, C.-W. Chou, and C.-H. Chu, "Evaluation of the efficacy and tolerability of miglitol in Chinese patients with type 2 diabetes mellitus inadequately controlled by diet and sulfonylureas," Acta Diabetol., vol. 48, no. 1, pp. 71-77, Mar. 2011, doi: 10.1007/s00592-010-0220-6. 
[10] A. Y. W. Lee and S. S. M. Chung, "Contributions of polyol pathway to oxidative stress in diabetic cataract," FASEB J., vol. 13, no. 1, pp. 23-30, Jan. 1999, doi: 10.1096/fasebj.13.1.23.

[11] S. K. Srivastava, K. V. Ramana, and A. Bhatnagar, "Role of Aldose Reductase and Oxidative Damage in Diabetes and the Consequent Potential for Therapeutic Options," Endocr. Rev., vol. 26, no. 3, pp. 380-392, May 2005, doi: 10.1210/er.2004-0028.

[12] M. Oka and N. Kato, “Aldose Reductase Inhibitors," J. Enzym. Inhib., vol. 16, no. 6, pp. 465-473, Jan. 2001, doi: 10.1080/14756360127568.

[13] D. Iqbal, M. S. Khan, M. S. Khan, S. Ahmad, M. S. Hussain, and M. Ali, "Bioactivity guided fractionation and hypolipidemic property of a novel HMG-CoA reductase inhibitor from Ficus virens Ait," Lipids Health Dis., vol. 14, no. 1, p. 15, Dec. 2015, doi: 10.1186/s12944-015-0013-6.

[14] Z. S. Derewenda, "Structure and Function of Lipases," in Advances in Protein Chemistry, vol. 45, C. B. Anfinsen, J. T. Edsall, F. M. Richards, and D. S. Eisenberg, Eds. Academic Press, 1994, pp. $1-52$.

[15] L.-F. Liang et al., "Brominated polyunsaturated lipids from the Chinese sponge Xestospongia testudinaria as a new class of pancreatic lipase inhibitors," Eur. J. Med. Chem., vol. 79, pp. 290297, May 2014, doi: 10.1016/j.ejmech.2014.04.003.

[16] I.-S. Lee, I. S. Kim, Y. M. Lee, Y. Lee, J.-H. Kim, and J. S. Kim, "2",4"-O-Diacetylquercitrin, a Novel Advanced Glycation End-Product Formation and Aldose Reductase Inhibitor from Melastoma sanguineum," Chem. Pharm. Bull. (Tokyo), vol. 61, no. 6, pp. 662-665, 2013, doi: 10.1248/cpb.c12-00877.

[17] B. Anitha Gopal and G. Muralikrishna, "Porcine Pancreatic $\alpha$-Amylase and its Isoforms: Purification and Kinetic Studies," Int. J. Food Prop., vol. 12, no. 3, pp. 571-586, May 2009, doi: 10.1080/10942910801947755.

[18] M. I. Kazeem, J. O. Adamson, and I. A. Ogunwande, "Modes of Inhibition of $\alpha$-Amylase and $\alpha$ -Glucosidase by Aqueous Extract of Morinda lucida Benth Leaf," BioMed Res. Int., vol. 2013, pp. 1-6, 2013, doi: 10.1155/2013/527570.

[19] V. Kumar, O. Prakash, S. Kumar, and S. Narwal, " $\alpha$-glucosidase inhibitors from plants: A natural approach to treat diabetes," Pharmacogn. Rev., vol. 5, no. 9, p. 19, 2011, doi: 10.4103/0973-7847.79096.

[20] M. S. Akhtar, M. K. Swamy, and U. R. Sinniah, Eds., Natural Bio-active Compounds: Volume 1: Production and Applications. Singapore: Springer Singapore, 2019.

[21] B. Lorenzati, C. Zucco, S. Miglietta, F. Lamberti, and G. Bruno, “Oral Hypoglycemic Drugs: Pathophysiological Basis of Their Mechanism of ActionOral Hypoglycemic Drugs: Pathophysiological Basis of Their Mechanism of Action," Pharmaceuticals, vol. 3, no. 9, pp. 3005-3020, Sep. 2010, doi: 10.3390/ph3093005.

[22] A. Mohammed, V. A. Gbonjubola, N. A. Koorbanally, and Md. S. Islam, "Inhibition of key enzymes linked to type 2 diabetes by compounds isolated from Aframomum melegueta fruit," Pharm. Biol., vol. 55, no. 1, pp. 1010-1016, Jan. 2017, doi: 10.1080/13880209.2017.1286358.

[23] S. Ponnusamy, S. Haldar, F. Mulani, S. Zinjarde, H. Thulasiram, and A. RaviKumar, "Gedunin and Azadiradione: Human Pancreatic Alpha-Amylase Inhibiting Limonoids from Neem (Azadirachta indica) as Anti-Diabetic Agents," PLOS ONE, vol. 10, no. 10, p. e0140113, Oct. 2015, doi: 10.1371/journal.pone.0140113. 
[24] Q. Abbas et al., "In vitro , in vivo and in silico anti-hyperglycemic inhibition by sinigrin," Asian Pac. J. Trop. Med., vol. 10, no. 4, pp. 372-379, Apr. 2017, doi: 10.1016/j.apjtm.2017.03.019.

[25] D. Ratna Wulan, E. Priyo Utomo, and C. Mahdi, "Antidiabetic Activity of Ruellia tuberosa L., Role of $\alpha$-Amylase Inhibitor: In Silico, In Vitro, and In Vivo Approaches," Biochem. Res. Int., vol. 2015, pp. 1-9, 2015, doi: 10.1155/2015/349261.

[26] C. Yh, J.-F. Hsieh, and S.-H. Wu, "Isolation and characterization of $\alpha$-glucosidase inhibitory constituents from Rhodiola crenulata," Food Res. Int. Ott. Ont, vol. 57, pp. 8-14, Mar. 2014, doi: 10.1016/j.foodres.2014.01.029.

[27] L. Zhang, B. Bai, X. Liu, Y. Wang, M. Li, and D. Zhao, “ $\alpha$-Glucosidase inhibitors from Chinese Yam (Dioscorea opposita Thunb.)," Food Chem., vol. 126, no. 1, pp. 203-206, May 2011, doi: 10.1016/j.foodchem.2010.10.100.

[28] H. W. Ryu et al., "Polyphenols from Broussonetia papyrifera Displaying Potent $\alpha$-Glucosidase Inhibition," J. Agric. Food Chem., vol. 58, no. 1, pp. 202-208, Jan. 2010, doi: 10.1021/jf903068k.

[29] K. Wang et al., "A novel class of $\alpha$-glucosidase and HMG-CoA reductase inhibitors from Ganoderma leucocontextum and the anti-diabetic properties of ganomycin I in KK-A y mice," Eur. J. Med. Chem., vol. 127, pp. 1035-1046, Feb. 2017, doi: 10.1016/j.ejmech.2016.11.015.

[30] M.-P. Dréanic, C. M. Edge, and T. Tuttle, "New Insights into the Catalytic Mechanism of Aldose Reductase: A QM/MM Study," ACS Omega, vol. 2, no. 9, pp. 5737-5747, Sep. 2017, doi: 10.1021/acsomega.7b00815.

[31] M. Y. Ali, H. A. Jung, S. Jannat, and J. S. Choi, "Dihydroxanthyletin-type coumarins from Angelica decursiva that inhibits the formation of advanced glycation end products and human recombinant aldose reductase," Arch. Pharm. Res., vol. 41, no. 2, pp. 196-207, Feb. 2018, doi: 10.1007/s12272-017-0999-6.

[32] G. B. Reddy, P. Muthenna, C. Akileshwari, M. Saraswat, and J. M. Petrash, "Inhibition of aldose reductase and sorbitol accumulation by dietary rutin," Curr. Sci., vol. 101, no. 9, p. 8, 2011.

[33] C. Akileshwari et al., "Bioflavonoid ellagic acid inhibits aldose reductase: Implications for prevention of diabetic complications," J. Funct. Foods, vol. 6, pp. 374-383, Jan. 2014, doi: 10.1016/j.jff.2013.11.004.

[34] P. Muthenna, P. Suryanarayana, S. K. Gunda, J. M. Petrash, and G. B. Reddy, "Inhibition of aldose reductase by dietary antioxidant curcumin: Mechanism of inhibition, specificity and significance," FEBS Lett., vol. 583, no. 22, pp. 3637-3642, Nov. 2009, doi: 10.1016/j.febslet.2009.10.042.

[35] Z. Wang, Y. N. Guillen Quispe, S. H. Hwang, G. Zuo, and S. S. Lim, "Pistafolin B is the major aldose reductase inhibitor of the pods of tara [Caesalpinia spinose (Molina) Kuntze]," Ind. Crops Prod., vol. 122, pp. 709-715, Oct. 2018, doi: 10.1016/j.indcrop.2018.06.023.

[36] H. Lee, "Inhibitory activity of Cinnamomum cassia bark-derived component against rat lens aldose reductase," J Pharm Pharm Sci, vol. 5, no. 3, pp. 226-30, 2002.

[37] S. S. Lim, Y. J. Jung, S. K. Hyun, Y. S. Lee, and J. S. Choi, "Rat lens aldose reductase inhibitory constituents of Nelumbo nucifera stamens: RAT LENS ALDOLASE AND NELUMBO NUCIFERA," Phytother. Res., vol. 20, no. 10, pp. 825-830, Oct. 2006, doi: 10.1002/ptr.1847. 
[38] P. Slanc, B. Doljak, S. Kreft, M. Lunder, D. Janeš, and B. Štrukelj, "Screening of selected food and medicinal plant extracts for pancreatic lipase inhibition," Phytother. Res., vol. 23, no. 6, pp. 874-877, Jun. 2009, doi: 10.1002/ptr.2718.

[39] P. Banerjee, A. O. Eckert, A. K. Schrey, and R. Preissner, "ProTox-II: a webserver for the prediction of toxicity of chemicals," Nucleic Acids Res., vol. 46, no. W1, pp. W257-W263, 02 2018, doi: 10/gdds4x.

[40] W. I. T. Fernando et al., "Isolation, identification and characterization of pancreatic lipase inhibitors from Trigonella foenum-graecum seeds," South Afr. J. Bot., vol. 121, pp. 418-421, Mar. 2019, doi: 10.1016/j.sajb.2018.10.023.

[41] G.-Y. Xia et al., "Three new polyoxygenated bergamotanes from the endophytic fungus Penicillium purpurogenum IMM 003 and their inhibitory activity against pancreatic lipase," Chin. J. Nat. Med., vol. 18, no. 1, pp. 75-80, Jan. 2020, doi: 10.1016/S1875-5364(20)30007-8.

[42] R. A. El-shiekh, D. A. Al-Mahdy, M. S. Hifnawy, and E. A. Abdel-Sattar, "In-vitro screening of selected traditional medicinal plants for their anti-obesity and anti-oxidant activities," South Afr. J. Bot., vol. 123, pp. 43-50, Jul. 2019, doi: 10.1016/j.sajb.2019.01.022.

[43] E. Komaki et al., "Identification of Anti-.ALPHA.-Amylase Components from Olive Leaf Extracts," Food Sci. Technol. Res., vol. 9, no. 1, pp. 35-39, 2003, doi: 10.3136/fstr.9.35.

[44] A. R. Abu Bakar, T. Manaharan, A. F. Merican, and S. B. Mohamad, "Experimental and computational approaches to reveal the potential of Ficus deltoidea leaves extract as $\alpha$-amylase inhibitor," Nat. Prod. Res., vol. 32, no. 4, pp. 473-476, Feb. 2018, doi: 10.1080/14786419.2017.1312393.

[45] F. Hichri, A. Omri, A. S. M. Hossan, and H. Ben Jannet, "Alpha-glucosidase and amylase inhibitory effects of Eruca vesicaria subsp. longirostris essential oils: synthesis of new 1,2,4-triazole-thiol derivatives and 1,3,4-thiadiazole with potential inhibitory activity," Pharm. Biol., vol. 57, no. 1, pp. 564-570, Jan. 2019, doi: 10.1080/13880209.2019.1642363.

[46] H.-Y. Ma, H.-Y. Gao, L. Sun, J. Huang, X.-M. Xu, and L.-J. Wu, “Constituents with $\alpha$-glucosidase and advanced glycation end-product formation inhibitory activities from Salvia miltiorrhiza Bge.," J. Nat. Med., vol. 65, no. 1, pp. 37-42, Jan. 2011, doi: 10.1007/s11418-010-0453-2.

[47] F. Moradi-Afrapoli et al., "In vitro $\alpha$-glucosidase inhibitory activity of phenolic constituents from aerial parts of Polygonum hyrcanicum," DARU J. Pharm. Sci., vol. 20, no. 1, p. 37, Dec. 2012, doi: 10.1186/2008-2231-20-37. 